\title{
Human Development Inequality Index and Cancer Pattern: a Global Distributive Study
}

\author{
Shahab Rezaeian ${ }^{1}$, Salman Khazaei ${ }^{2}$, Somayeh Khazaei ${ }^{3}$, Kamyar Mansori ${ }^{4}$, Ali \\ Sanjari Moghaddam ${ }^{5}$, Erfan Ayubi ${ }^{6,7 *}$
}

\begin{abstract}
This study aimed to quantify associations of the human development inequality (HDI) index with incidence, mortality, and mortality to incidence ratios for eight common cancers among different countries. In this ecological study, data about incidence and mortality rates of cancers was obtained from the Global Cancer Project for 169 countries. HDI indices for the same countries was obtained from the United Nations Development Program (UNDP) database. The concentration index was defined as the covariance between cumulative percentage of cancer indicators (incidence, mortality and mortality to incidence ratio) and the cumulative percentage of economic indicators (country economic rank). Results indicated that incidences of cancers of liver, cervix and esophagus were mainly concentrated in countries with a low HDI index while cancers of lung, breast, colorectum, prostate and stomach were concentrated mainly in countries with a high HDI index. The same pattern was observed for mortality from cancer except for prostate cancer that was more concentrated in countries with a low HDI index. Higher MIRs for all cancers were more concentrated in countries with a low HDI index. It was concluded that patterns of cancer occurrence correlate with care disparities at the country level.
\end{abstract}

Keywords: Concentration index - mortality to incidence ratio - cancer - human development index

Asian Pac J Cancer Prev, 17, Cancer Control in Western Asia Special Issue, 201-204

\section{Introduction}

Cancer is one of the leading causes of death among women population worldwide (Jemal et al., 2011). It is estimated that cancer led to 13.2 million deaths with 20.3 million new cases by 2030 compared with an estimated 7.6 million deaths and 12.7 million cases in 2008 (Ferlay et al., 2010). Over the past decades, the global patterns of cancer incidence and mortality have been changed. The highest incidence and mortality rates of many cancers were found in more developed countries compared to less developed countries (Porter, 2009; Jemal et al., 2010; Youlden et al., 2012). Recently, one novel measure named Mortality to Incidence Ratio (MIR) is introduced that can be used to evaluate cancer mortality in relation to incidence. MIR as proxy of survival can be a more sensitive measure to availability cancer care including screening, diagnosis and treatment previous studies found that developed countries e.g. strong national health system had lower than expected MIR (Hébert et al., 2009; Sunkara and Hébert, 2015). Several studies showed that the incidence and mortality disparities between countries can be attributed to some factors such as differences in life expectancy, education level, income level, and access to healthcare (Coughlin et al., 2007; Akinyemiju, 2012; Myers et al., 2015). Human Development Inequality (HDI Index as a key socioeconomic determinant of health is composite of three components including education, life expectancy, and gross national income. The relation of different cancers and levels of HDI at national and sub national level is studied and a possible inverse association is found (Patel et al., 2012). To achieve a clear judgment about a degree socioeconomic inequality in health variables more sensitive measures such as concentration index is needed. Concentration index is constant to multiplication of the health variable by any scalar and whether health variable is measured in terms per month or year. Using the concentration index to clarify the HDI on cancer measure, the aim was assess the HDI inequalities in cancers measures including incidence, mortality and MIR four eight common cancers with worldwide aggregated data.

${ }^{1}$ Social Development \& Health Promotion Research Center, Gonabad University of Medical Sciences, Gonabad, ${ }^{2}$ Department of Biostatistics and Epidemiology, School of Public Health, Hamadan University of Medical Sciences, Hamadan, ${ }^{3}$ Rafsanjan University of Medical Sciences, Rafsanjan, ${ }^{4}$ Department of Epidemiology, School of Medicine, Kurdistan University of Medical Sciences, Sanandaj, ${ }^{5}$ School of Medicine, ${ }^{6}$ School of Public Health, Shahid Beheshti University of Medical Sciences, ${ }^{7}$ School of Public Health, Tehran University of Medical Sciences, Tehran, Iran, *For correspondence: aubi65@gmail.com 


\section{Material and Methods}

\section{Study design}

Present ecological study used dataset regarding the incidence and mortality rates of eight common cancers and HDI in the world. Data of the incidence and mortality rates of cancers was obtained from the global cancer project for 172 countries in year 2012. GLOBOCAN also provided regional estimates for each continent [available at http:// globocan.iarc.fr/Default.aspx]. Data about the HDI 2013 were obtained from the United Nations Development Programme (UNDP) database for 169 countries [available at http://databank.worldbank.org/data/reports.aspx]

\section{Measures}

The data of three countries due to missing observations in some variables were excluded. Hence, the analysis was restricted to 169 countries. We defined inequality in the age-specific incidence and mortality rates (ASR) and mortality to incidence ratio (MIR) of eight common cancers related to the HDI inequality by using concentration index.

\section{Data analysis}

Concentration index provide a way to assess the degree of health related economic inequality. The concentration index defines as the covariance between cumulative percentage of health variable (cancer measure) and the cumulative percentage of economic variable as follow:

$$
\mathrm{C}=\frac{2}{\mu} \operatorname{cov}\left(h_{i}, r_{i}\right)
$$

Where hi is the health variable, $\mu$ is its mean, and $\mathrm{ri}=$ $\mathrm{i} / \mathrm{N}$ is the fractional rank of individual $\mathrm{i}$ in the economic, with $\mathrm{i}=1$ for the poorest and $\mathrm{i}=\mathrm{N}$ for the richest.

The value of concentration index is ranged from -1 to +1 , the negative value is indicating that the health variable is more concentrated in the poor population (pro-poor) and the positive value indicates in rich population (pro- rich).

Data were analyzed by Distributive Analysis Stata Package (DASP) in Stata software version 12 (StataCorp, College Station, TX, USA).

\section{Results}

Of new cancer cases estimated in 2012, more than
$60 \%$ incident cases occurred as result of eight common cancers and lung cancer had the most proportion (13\%). Incidence and mortality geographic inequality of each cancer varied in developed and under developed countries by HDI so that most of cancer incidence was concentrated in developed countries. MIR for all eight cancers was concentrated in under developed countries. Concentration index and $\% 95$ confidence interval for incidence, mortality and MIR is presented in detail in Table 1.

\section{Discussion}

This study indicates that cancers incidence of liver, cervical and esophagus has become more concentrated in low HDI countries and in contrast, cancers incidence of lung, breast, colorectal, prostate, and stomach are more concentrated in developed countries by HDI. This pattern was consistent for mortality expect for prostate cancer, it is more concentrated in low HDI countries. Geographic disparity of cancer MIR have been aggravate so that for all studied cancers it was more concentrated in low HDI countries.

It has been suggested that evidence about potential association between macroeconomic determinants and cancer incidence, mortality, and survival is lacking. One of the best attempts to fill this gap was a study that aimed to clarify the potential association of cancer burden measures against HDI. It found that in high HDI regions four cancers (breast, lung, colorectal and prostate) explain half the overall cancer burden whereas in low HDI regions four mentioned cancer along with esophagus, stomach, and liver cancers account for about $62 \%$ of the burden (Bray et al., 2012).

The more concentration cancers incidence in developed countries may likely reflect higher rates of screening programs, diagnostic modalities or even high quality population based cancer registries that lead to cancers more likely to be diagnosed (Hébert et al., 2009). One important reason to high concentration of cervical cancer incidence in less developed regions is high prevalence of infection with human papillomavirus (HPV) in these regions, in addition it shows in low HDI countries screening program for cervical cancer is less likely to have been with highly effectiveness (Forman et al., 2012). It is

Table 1. Concentration Index (95\% CI) for HDI Inequality in Incidence Rate, Mortality Rate and Mortality to Incidence Ratio of Eight Cancers

\begin{tabular}{lccccc}
\hline Cancer type & $\begin{array}{c}\text { new diagnosis cases } \\
(\%) \text { in 2012 }(1,000 \mathrm{~s}) \\
\text { Total incidence } \\
\text { cases=14,067,894 }\end{array}$ & $\begin{array}{c}\text { New death } \\
\text { cases }(\%) \text { in 2012 } \\
(1,000 \mathrm{~s}) \text { Total } \\
\text { death case= } \\
8,201,575\end{array}$ & $\begin{array}{c}\text { Incidence rate } \\
\text { concentration index } \\
(95 \% \mathrm{CI})\end{array}$ & $\begin{array}{c}\text { Mortality rate } \\
\text { concentration index } \\
(95 \% \mathrm{CI})\end{array}$ & $\begin{array}{c}\text { Mortality to } \\
\text { Incidence Ratio } \\
\text { concentration index } \\
(95 \% \mathrm{CI})\end{array}$ \\
\hline Lung & $1,825.0(13.0)$ & $1,590.0(19.4)$ & $0.29(0.23,0.35)$ & $0.27(0.22,0.33)$ & $-0.02(-0.02,-0.01)$ \\
Breast & $1,677.0(11.9)$ & $521.0(6.3)$ & $0.24(0.22,0.28)$ & $0.05(0.03,0.08)$ & $-0.17(-0.19,-0.15)$ \\
Colorectal & $1,361.0(9.7)$ & $693.0(8.4)$ & $0.3(0.26,0.34$ & $0.18(0.15,0.22)$ & $-0.12(-0.14,-0.1)$ \\
Prostate & $1,112.0(7.9)$ & $307.0(3.7)$ & $0.28(0.22,0.34)$ & $-0.04(-0.09,0.01)$ & $-0.22(-0.26,-0.18)$ \\
Stomach & $952.0(6.8)$ & $723.0(8.8)$ & $0.08(0.02,0.14)$ & $0.01(-0.04,0.06)$ & $-0.07(-0.08,-0.05)$ \\
Liver & $782.0(5.6)$ & $746.0(9.1)$ & $-0.1(-0.17,-0.027)$ & $-0.1(-0.17,-0.03)$ & $-0.14(-0.18,-0.1)$ \\
Cervical & $528.0(3.7)$ & $266.0(3.2)$ & $-0.2(-0.25,-0.15)$ & $-0.3(-0.38,-0.25)$ & $-0.13(-0.16,-0.1)$ \\
Esophagus & $456.0(3.2)$ & $400.0(4.8)$ & $-0.23(-0.33,-0.13)$ & $-0.25(-0.36,-0.15)$ & $-0.03(-0.04,-0.01)$ \\
\hline
\end{tabular}


argued that HDI transitions lead to changes in the scale and profile of cancer occurrence, e.g. it has identified that in countries that have a transition towards higher levels of human development (westernization effect) rapid declines in the incidence of cervical cancer offset by concurrent increases in female breast cancer (Bray et al., 2012). It can be pointed that the two cancers intersect is a marker of the extent of economic transition in a given country.

MIR as surrogate of cancer care disparities and cancer survival (Hébert et al., 2009; Vostakolaei et al., 2011) can be used to evaluate cancer mortality in relation to incidence. In one global study in Economic Cooperation and Development (OECD) countries using the 2012 GLOBOCAN incidence and mortality statistics, investigators argued that MIR can be indicator of cancer screening and treatment internationally, in addition they found low HDI countries that have lower health system ranked by world health organization (WHO) the higher colorectal cancer MIR is observed (Sunkara and Hébert, 2015). A consistent finding with former study, our study showed that colorectal cancer MIR more concentrated in low HDI countries. The more concentration of colorectal cancer MIR in under developed countries may probably explained by expensive treatment when diagnosed at a later stage and in following a poorer survival.

We found MIR of gastrointestinal cancers overall is concentrated in low HDI countries, a finding consistent with other global study (Hu et al., 2013). In that global study it has been proposed MIR of all gastrointestinal cancers inversely is related with HDI. Present study suggested in specific, the incidence and mortality of colorectum and stomach cancers have been concentrated in high HDI regions but for esophagus cancer in low HDI regions. However important point is mentioned association not have been constrain to gastrointestinal cancers because other evidence suggest that given MIR is related inversely with HDI, burden of different cancers disparity would be worsen in developing countries (Patel et al., 2012). For lung, breast, colorectum, and stomach cancers the incidence and mortality are concentrated in developed countries but MIR in under developed countries. One reason to this come back to matter of MIR that is a proxy of survival.

In a global study that have been looked to whether all cancer MIR is related to country's income level by Gross Domestic Product (GDP). They categorized countries by GDP $>\$ 15,000$ and GDP $<\$ 15,000$ as high-income and middle/low-income respectively, their results shown an inverse association between MIR and GDP so that cancer MIR was higher in middle/low-income countries. In addition their finding suggested that for middle/ low-income countries, given an inequality in healthcare resources, increased health care expenditure may not associated with improved national cancer MIR (Batouli et al., 2014). To achieve valid results all-inclusive indicators should be applied, here HDI including education, health, and living standards more than per capita GDP express complex profile of society development.

Our results showed that lung cancer MIR approached to equality line. It suggested that lung cancer MIR can raise above 0.80 and approached to 1 , this can be as result of high fatality and relative lack of variability in survival in different world regions (Kamangar et al., 2006; Hébert et al., 2009). Our finding suggest lung cancer incidence and mortality have nearly same concentration index, it means the geographical patterns in mortality closely follow those in incidence. In present study, also equality line and same CI of incidence and mortality were observed for esophageal cancer. An international study showed overall cancer incidence rates of esophageal are higher in less developed compared with more-developed geographic regions and because of poor survival the mortality and incidence close together (Kamangar et al., 2006).

The important limitation of present study should be considered, ecological studies are potentially susceptible to a phenomenon as "ecological fallacy"; biases that may occur when an observed association between aggregated variables differs from the true. Here the association between cancer measures and HDI may have a different patterns and interpretations within countries, anyway our finding can be considered as extends knowledge by different methodology in following already known evidences. Our result showed that MIR for all studied cancers are more concentrated in low HDI countries. This conclusion should be interpreted with caution because cancer is a notorious multi-causal multi-phase chronic diseases. According to gene-environmental interaction, only economic terms cannot explain the statistics even if MIR might be a proxy of survival. Moreover to achieve the fine these global inequality by HDI, it's needed the concentration index to be decomposed, which component of HDI have most contribution in these inequality and geographic disparity.

In summary, our distributive analysis shown cancer MIR as more sensitive measure to geographic disparity, cancer care disparity and survival is more concentrated in underdeveloped countries by HDI. Clarifying the contributions proportion of these HDI inequality in cancer measures globally and even within a given country is likely an appropriate goal to further knowledge.

\section{Acknowledgements}

This paper used data from the GLOBOCAN and the United Nations Development Programme (UNDP). The authors declare that there is no conflict of interests to report for this work.

\section{References}

Akinyemiju TF (2012). Socio-economic and health access determinants of breast and cervical cancer screening in low-income countries: analysis of the World Health Survey. PLoS One, 7, 4883-4.

Batouli A, Jahanshahi P, Gross CP, et al (2014). The global cancer divide: Relationships between national healthcare resources and cancer outcomes in high-income vs. middle-and low-income countries. J Epidemiol Glob Health, 4, 115-24.

Bray F, Jemal A, Grey N, et al (2012). Global cancer transitions according to the human development index (2008-2030): a population-based study. Lancet Oncol, 13, 790-801.

Coughlin SS, Berkowitz Z, Hawkins NA, et al (2007). Breast and colorectal cancer screening and sources of cancer 
information among older women in the united states: results from the 2003 health information national trends survey. Prev Chronic Dis, 4, A57.

Ferlay J, Shin HR, Bray F, et al (2010). Estimates of worldwide burden of cancer in 2008: Globocan 2008. Int J Cancer, 127, 2893-917.

Forman D, de Martel C, Lacey CJ, et al (2012). Global burden of human papillomavirus and related diseases. Vaccine, $\mathbf{3 0}$, F12-F23.

Hébert JR, Daguise VG, Hurley DM, et al (2009). Mapping cancer mortality to incidence ratios to illustrate racial and sex disparities in a high-risk population. Cancer, 115, 2539-52.

$\mathrm{Hu}$ Q-D, Zhang Q, Chen W, et al (2013). Human development index is associated with mortality-to-incidence ratios of gastrointestinal cancers. World J Gastroenterol, 19, 5261 .

Jemal A, Bray F, Center MM, et al (2011). Global Cancer Statistics. CA Cancer J Clin, 61, 69-90.

Jemal A, Center MM, DeSantis C, et al (2010). Global patterns of cancer incidence and mortality rates and trends. Cancer Epidemiol Biomarkers Prev, 19, 1893-907.

Kamangar F, Dores GM, Anderson WF (2006). Patterns of cancer incidence, mortality, and prevalence across five continents: defining priorities to reduce cancer disparities in different geographic regions of the world. J Clin Oncol, 24, 2137-50.

Myers ER, Moorman P, Gierisch JM, et al (2015). Benefits and harms of breast cancer screening: a systematic review. JAMA, 314, 1615-34.

Patel AR, Prasad SM, Shih Y-CT, et al (2012). The association of the human development index with global kidney cancer incidence and mortality. J Urol, 187, 1978-83.

Porter PL (2009). Global trends in breast cancer incidence and mortality. Salud Publica Mex, 51, 141-6.

Sunkara V, Hébert JR (2015). The colorectal cancer mortality to incidence ratio as an indicator of global cancer screening and care. Cancer, 121, 1563-9.

Vostakolaei FA, Karim-Kos HE, Janssen-Heijnen ML, et al (2011). The validity of the mortality to incidence ratio as a proxy for site-specific cancer survival. Eur J Public Health, 21, 573-7.

Youlden DR, Cramb SM, Dunn NA, et al (2012). The descriptive epidemiology of female breast cancer: an international comparison of screening, incidence, survival and mortality. Cancer Epidemiol, 36, 237-48. 